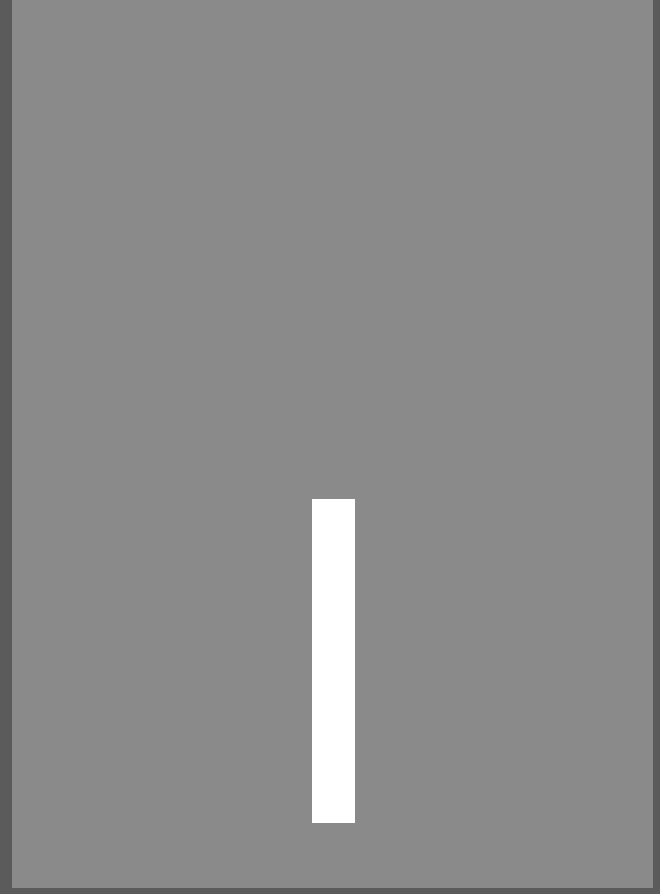

\title{
PRODUCCIÓN INTELECTUAL Y VISIBILIDAD CIENTÍFICA
}

\author{
Intellectual production and scientific visibility \\ Universidad Peruana Unión, Perú
}

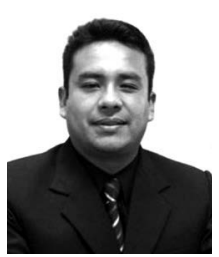

\section{Josué E. Turpo}

Licenciado en Teología por la Universidad Peruana Unión. Magíster en Educación por la Universidad Peruana Unión. Doctorando en Educación por la Universidad Peruana Unión. Docente asociado y catedrático de investigación en Ciencias Sociales en la Universidad Peruana Unión. Director de Investigación de la Universidad Peruana Unión, Tarapoto. Su investigación se centra en la educación organizacional, educación integral y hermenéutica. Director de la revista de investigación Apuntes Universitarios Ha publicado artículos en revistas como Internacional de Estudios en Educación y Apuntes Universitarios.

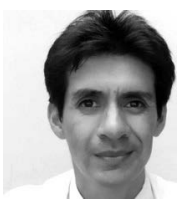

\section{Gerardo E. Medina}

Licenciado en Educación por la Universidad Peruana Unión. Maestrando en Administración y Recursos Humanos por la Universidad Nacional del Altiplano. Docente asociado de la cátedra de lingüística de la Universidad Peruana Unión. Coordinador de los programas gubernamentales Beca 18 de la Universidad Peruana Unión. Ponente y docente visitante. 


\section{Resumen}

Una de las dimensiones exigida por las agencias acreditadoras a las universidades es que haya producción de conocimiento. El último informe SIR 2013 (grupo SClmago), muestra que las universidades latinoamericanas se encuentran relegadas en investigación en comparación con las universidades estadounidenses o europeas, al mismo tiempo señala que la investigación en el Perú es deficiente, y más aún en las universidades confesionales. Basándonos en la premisa de que "La ciencia que no se ve, no existe" y que las nuevas normatividades exigen realizar producción intelectual, es necesario analizar la producción científica y su relación con la visibilidad. El presente estudio narra la experiencia de la revista de investigación Apuntes Universitarios y de cómo se ha consolidado como la segunda revista con referato internacional de la Universidad Peruana Unión; al mismo tiempo explica los pasos que la llevaron a estar incluida en los catálogos de revistas más importantes de Latinoamérica.

Palabras clave: Producción científica; visibilidad; ranking universitario.

\section{Abstract}

One of the dimensions required by accrediting agencies to universities is that no production of knowledge. The latest 2013 SIR (SCImago group) shows that Latin American universities are confined in research compared to U.S. or European universities at the same time notes that research in Peru is poor, and more so in the universities confessional. Based on the premise that "the science is not there " and that new intellectual production normativities which warrant is necessary to analyze the scientific production and its relation to visibility. This study recounts the experience of the Journal of College and Research Notes how it has become the second international refereed journal Peruvian Union University, at the same time explains the steps taken to be included in the catalogs of magazines important in Latin America.

Keywords: Scientific production; visibility; university ranking. 


\section{Introducción}

La investigación es una de las áreas más importantes de toda institución universitaria (Korniejczuck, 2005), la misma que debe estar fundamentada en la publicación científica. De allí que investigación sin publicación no es investigación, o como declara el dicho "La ciencia que no se ve, no existe" (REDALYC 2013). Más aún, en el entorno universitario en que se vive, es necesario facilitar la interacción entre la sociedad del conocimiento (Pacheco y Hurtado, 2012) y nuestros investigadores, lo que evita no solo mayores recursos sino también la duplicidad de las investigaciones. Por ello es necesario comprender lo que significa visibilidad de la producción científica y la aplicación que podemos dar como universidad. Asimismo, para fines de acreditación universitaria y el posicionamiento en los rankings internacionales, es necesario conocer el impacto citacional de una investigación, lo que se logra gracias a la indexación.

\section{Importancia de la visibilidad científica}

La visibilidad científica está relacionada con la cantidad de materiales y publicaciones que produce una universidad y que pueden ser vistas por la comunidad científica. Para lograr la mayor visibilidad científica, necesitamos conocer los factores involucrados en el campo de la medición de la calidad científica (Ardito, 2012). Esta visibilidad se puede lograr: publicando una mayor cantidad de libros; creando una revista científica; adecuando nuestras revistas de acuerdo a los criterios de las bases de datos; creando políticas institucionales de incentivos para la publicación de manuscritos; capacitando a los investigadores en todos los procesos de publicación. Sea cual fuere el camino a tomar, se deben seguir políticas serias para incrementar esta visibilidad.

Una revista científica es una publicación que cuenta con una periodicidad establecida, con artículos inéditos, producto del resultados de investigación que son debidamente arbitrados por un comité editorial reconocido a nivel nacional e internacional y que, además, se apega estrictamente a la normativa establecida para las publicaciones, cuyo principal objetivo es comunicar ciencia" (Rojas y Rivera 2012:24). Asimismo, el impacto citacional representa el número de veces que ha sido citado el artículo por otros investigadores. Este área de estudios pertenece a la cienciometría o bibliometría, los cuales son tendientes a analizar el impacto que pueda tener un autor determinado en su entorno investigativo alrededor del mundo (Pacheco y Hurtado, 2012). 
Por otro lado, la indexación es la medida natural de la producción científica de calidad, permite no solo que la comunidad regional se entere de la investigación local, sino además permiten a los académicos e investigadores de la región puedan llegar a comunidades locales, nacionales y, gracias al Open Acces, a todo el mundo. Por ello la importancia para las universidades de contar con revistas indexadas, y lograr que sus docentes e investigadores publiquen en revistas científicas de alto impacto. Asimismo, se debe implementar prácticas en la cual los investigadores citen artículos de las revistas científicas institucionales, y entre ellas puedan ayudarse a elevar el nivel de visibilidad. Así también orientar a los investigadores que coloquen claramente el nombre de la universidad en la cual se desempeñan de forma unificada, en el caso nuestro podría ser Universidad Peruana Unión, Perú. También el fomento de incentivos que podría ser bonificaciones que van desde los US\$100, por capítulos de libros, hasta los US\$5000 por artículos publicados en revistas indexadas en ISI o Scopus, tal como lo hacen algunas universidades (Uniandes, 2006).

El concepto de indización está relacionado a la técnica de análisis de documentos, el cual tiene por objetivo representar y describir el contenido de los mismos, mediante conceptos principales contenidos en ellos (palabras clave) o vocabularios controlados (descriptores, términos o encabezamientos de materia), con la finalidad de guiar al usuario en la recuperación de documentos a los que necesita acceder; al mismo tiempo se debe hacer diferencia la palabra indización de la indexación, que es otro término muy frecuentemente utilizado, se entiende como el proceso por el cual el buscador va rastreando el sitio en cuestión y al mismo tiempo va incorporando a su base de datos el contenido de esas URLs, es decir, se registra ordenadamente información para elaborar un índice. Este procedimiento es de vital importancia para las páginas web, ya que es el que permite que la página web aparezca en alguno de los buscadores más importantes, aquella página que no se haya indexado como debería no aparecerá entre los resultados de una búsqueda, en palabras sencillas indexar es agregar una página sin importar en que formato se encuentre a la lista de resultados de un buscador (Aguirre, 2012). 
Las principales redes de indexación son:

\begin{tabular}{|c|c|c|c|}
\hline Índice & Descripción & $\begin{array}{l}\text { Artículos } \\
\text { de texto } \\
\text { completo }\end{array}$ & $\begin{array}{c}\text { Tipo de } \\
\text { acceso a los } \\
\text { artículos }\end{array}$ \\
\hline Dialnet & $\begin{array}{l}\text { Repositorio o biblioteca virtual y base de datos } \\
\text { especializada, creada en el año } 2001 \text { por la Uni- } \\
\text { versidad de La Rioja, España. Con predominio en } \\
\text { Ciencias Sociales y Humanidades, incluye también } \\
\text { revistas de Ciencia y Tecnología. Su objetivo es } \\
\text { aumentar la difusión, visibilidad y accesibilidad } \\
\text { de la literatura científica hispana, potenciando el } \\
\text { acceso libre y gratuito. Para conseguir ese objetivo, } \\
\text { Dialnet ofrece a los servicios de publicaciones de } \\
\text { entidades cuya actividad no tenga ánimo de lucro, } \\
\text { el alojamiento de los textos completos de sus pu- } \\
\text { blicaciones, tanto revistas como tesis doctorales } \\
\text { y libros colectivos. }\end{array}$ & $\mathrm{Si}$ & Gratuito \\
\hline Latindex & $\begin{array}{l}\text { Latindex creado en } 1997 \text { es conformado por } 19 \\
\text { organismos gubernamentales y académicos repre- } \\
\text { sentantes de distintos países latinoamericanos. Es } \\
\text { un directorio que recoge información sobre revis- } \\
\text { tas científicas, profesionales y técnicas. En el } 2002 \\
\text { se creó el Catálogo, que contiene una selección de } \\
\text { las revistas incluidas en el directorio de acuerdo a } \\
36 \text { criterios de calidad editorial internacional. El } \\
\text { Catálogo Latindex no indiza artículo por artículo, } \\
\text { sino que entrega información descriptiva sobre la } \\
\text { revista a nivel de título. }\end{array}$ & No & Gratuito \\
\hline $\begin{array}{l}\text { Clase (Citas } \\
\text { latinoameri- } \\
\text { canas } \\
\text { en Ciencias } \\
\text { Sociales y } \\
\text { Humanida- } \\
\text { des) }\end{array}$ & $\begin{array}{l}\text { CLASE y PERIÓDICA indizan revistas académicas } \\
\text { de investigación, técnico-profesionales y de di- } \\
\text { fusión científica o cultural, editadas en países de } \\
\text { América Latina y el Caribe. También se incluyen } \\
\text { revistas editadas por organismos internacionales } \\
\text { de alcance panamericano. Las revistas pueden ser } \\
\text { especializadas o multidisciplinarias, en formato } \\
\text { impreso o electrónico. Las revistas especializadas } \\
\text { en ciencias sociales y humanidades se indizan en } \\
\text { CLASE, mientras que las de ciencia y tecnología } \\
\text { se incluyen en PERIÓDICA. Las multidisciplinarias } \\
\text { pueden incluirse en alguna de las dos bases } \\
\text { de datos, conforme a la opinión del Comité de } \\
\text { Selección. }\end{array}$ & $\mathrm{Si}$ & Gratuito \\
\hline
\end{tabular}


Josué E. Turpo \& Gerardo E. Medina

\begin{tabular}{|c|c|c|c|}
\hline Índice & Descripción & $\begin{array}{l}\text { Artículos } \\
\text { de texto } \\
\text { completo }\end{array}$ & $\begin{array}{c}\text { Tipo de } \\
\text { acceso a los } \\
\text { artículos }\end{array}$ \\
\hline $\begin{array}{l}\text { Revistas } \\
\text { Peruanas }\end{array}$ & $\begin{array}{l}\text { Portal de Revistas Peruanas Científicas y Técnicas } \\
\text { bajo la administración del Consejo Nacional de } \\
\text { Ciencia y Tecnología del Perú, CONCYTEC-PERÚ. } \\
\text { Aquí se encuentran las revistas que siguen me- } \\
\text { todología Scielo, pero que todavía no han sido } \\
\text { evaluadas para su ingreso en Scielo. }\end{array}$ & $\mathrm{Si}$ & Gratuito \\
\hline $\begin{array}{l}\text { AJ (Directory } \\
\text { of open acces } \\
\text { journals) }\end{array}$ & $\begin{array}{l}\text { Directorio administrado y parcialmente financiado } \\
\text { por el sistema de Biblioteca de la Universidad de } \\
\text { Lund. Indexa solo revistas en línea de contenidos } \\
\text { abiertos. Dentro de sus requisitos está que los } \\
\text { contenidos sean totalmente accesibles, no acepta } \\
\text { embargos por períodos. Las revistas seleccionadas } \\
\text { son principalmente académicas y científicas, pone } \\
\text { como requisito que estén primariamente orienta- } \\
\text { das a un público conformado por investigadores. } \\
\text { No limita la postulación de revistas por idioma o } \\
\text { área temática. Su objetivo es incrementar la visibi- } \\
\text { lidad y la facilidad de uso de las revistas. }\end{array}$ & $\mathrm{Si}$ & Gratuito \\
\hline $\begin{array}{l}\text { Redalyc (Red } \\
\text { de revistas } \\
\text { científicas } \\
\text { de América } \\
\text { Latina y El Ca- } \\
\text { ribe, España y } \\
\text { Portugal) }\end{array}$ & $\begin{array}{l}\text { Creado en el } 2003 \text { por la Universidad Autónoma del } \\
\text { Estado de México. Indiza revistas principalmente } \\
\text { de América Latina, España y Portugal. Tiene una } \\
\text { amplia cobertura de las ciencias sociales. Las pos- } \\
\text { tulaciones al índice son en febrero y julio de cada } \\
\text { año. Observa } 39 \text { criterios de selección, de los cua- } \\
\text { les } 27 \text { son generales, } 12 \text { son básicos y } 5 \text { esenciales. } \\
\text { Para poder ser indizada, una revista debe cumplir } \\
\text { al menos } 20 \text { de los } 27 \text { criterios. Si una revista tiene } \\
\text { versión impresa y digital, se sugiere postular solo } \\
\text { una de las versiones. }\end{array}$ & $\mathrm{Si}$ & Gratuito \\
\hline $\begin{array}{c}\text { SCIELO } \\
\text { (Scientific } \\
\text { Electronic } \\
\text { Library On- } \\
\text { line) }\end{array}$ & $\begin{array}{l}\text { Creado por la Fundación de Apoyo a la Investigación } \\
\text { del Estado de São Paulo y por Centro Latinoameri- } \\
\text { cano y del Caribe de Información en Ciencias de la } \\
\text { Salud. Esta biblioteca electrónica que incluye una } \\
\text { colección seleccionada de revistas científicas. En } \\
\text { Perú es administrado por CONCYTEC. Para su acep- } \\
\text { tación las revistas son evaluadas según } 17 \text { criterios } \\
\text { centrados en la calidad de los artículos publicados, } \\
\text { que deben ser originales y en su mayoría de corte } \\
\text { científico y en la implementación de un proceso } \\
\text { de revisión por pares hecho por especialistas. Las } \\
\text { revistas que ingresen deben seguir rigurosamente } \\
\text { la metodología Scielo, así como el uso de metada- } \\
\text { tos y de referencias por separado. Las revistas son } \\
\text { aceptadas de forma inmediata si están incluidas en } \\
\text { Thompson Reuters (ex ISI), MEDLINE o PsycINFO. }\end{array}$ & $\mathrm{Si}$ & Gratuito \\
\hline
\end{tabular}




\begin{tabular}{|c|l|c|c|}
\hline Índice & \multicolumn{1}{|c|}{ Descripción } & $\begin{array}{c}\text { Artículos } \\
\text { de texto } \\
\text { completo }\end{array}$ & $\begin{array}{c}\text { Tipo de } \\
\text { acceso a los } \\
\text { artículos }\end{array}$ \\
\hline SCOPUS & $\begin{array}{l}\text { Creado el año 2004 por la empresa Elsevier, esta } \\
\text { base de datos cubre más de 27 000 revistas. Cuen- } \\
\text { ta con 420 títulos latinoamericanos y al menos } \\
\text { un 21\% de los títulos cubiertos en Scopus están } \\
\text { en idiomas diferentes al inglés. Para evaluar la } \\
\text { inclusión de nuevas revistas observa 16 criterios, } \\
\text { agrupados en 5 categorías: política editorial (in- } \\
\text { cluye revisión por pares modalidad doble ciego } \\
\text { y diversidad de autores y de comité editorial), } \\
\text { contenido (académico y relevante al área de la re- } \\
\text { vista), provisión de referencias bibliográficas y citas } \\
\text { correctas y bien estructuradas; y periodicidad. Es } \\
\text { un indicador bibliométrico en que se añaden títulos } \\
\text { nuevos a Scopus dos veces al año: enero (para los } \\
\text { títulos enviados antes del 1 de setiembre del año } \\
\text { anterior) y julio (para los títulos enviados antes del } \\
\text { 1 de marzo del presente año). }\end{array}$ & Suscripción \\
\hline $\begin{array}{c}\text { Web of Scien- } \\
\text { ce y Web of } \\
\text { nowledge: } \\
\text { ISI (Institute } \\
\text { of Scientific }\end{array}$ & $\begin{array}{l}\text { Fue adquirido el año 2002 por Thompson Reuters. } \\
\text { Esta empresa es la encargada de elaborar los índi- } \\
\text { ces Web of Science. Contiene las revistas científicas } \\
\text { consideradas más prestigiosas por su factor de im- } \\
\text { pacto a nivel global, y Web of Knowledge. Además } \\
\text { de indizar revistas de ciencia también incorpora } \\
\text { títulos de ciencias sociales, artes y humanidades. } \\
\text { La selección de revistas está basada en la perio- } \\
\text { dicidad; en que la revista publique información } \\
\text { bibliográfica en inglés; revisión por pares y tener } \\
\text { un formato que asegure la correcta citación de los } \\
\text { artículos publicados. Este índice se ha convertido } \\
\text { en el indicador más reconocido de calidad editorial } \\
\text { de las revistas y de bibliometría. }\end{array}$ & No & Suscripción \\
\hline & & \\
\hline
\end{tabular}

Fuente: Iturrieta (2012) Sugerencias para la indexación de revistas de trabajo social como espacios de visibilidad de la productividad académica. Adaptado.

\section{Revista Apuntes Universitarios}

Las universidades peruanas se encuentran relegadas en los rankings internacionales (SIR, 2013) - donde prima la investigación y las publicaciones en revistas con alto impacto-. La Universidad Peruana Unión es una universidad privada confesional, con 30 años de vida universitaria, se encuentra entre las universidades privadas más representativas (Top universities 2013; Pisco- 
ya, 2006), sin embargo, al igual que sus pares, en los rankings internacionales (Sir Scimagor) su presencia es casi nula, a no ser por la presencia de algunos investigadores que realizaron posgrados en el extranjero y participan en redes científicas internacionales. Gregorutti (2010; 2011), realizó estudios relacionados a la producción científica de las universidades privadas confesionales, concluyendo que se deben crear políticas de cambio que incrementen la producción intelectual, y aunque históricamente las instituciones universitarias adventistas no le han dado mucho énfasis a la investigación (Greenleaf, 2009. pp. 368, 369. Martin y Wilson, 2002.), sin embargo, se pueden y deben hacer mejoras, especialmente en otorgar los fondos y recursos necesarios. Existen sin embargo, algunas excepciones como es el caso de la revista THEOLOGIKA, la que se encuentra en catálogos internacionales y posee un nivel ganado en los últimos 30 años; sin embargo, como institución no se contaba con una revista bandera que pudiera ingresar en las redes de indexación y bases de datos logrando una mayor visibilidad de los investigadores, permitiéndoles salir del anonimato y llevar sus investigaciones a la palestra.

Por otro lado, si un investigador desea publicar en una revista internacional que se encuentre en la Web Knowles o en el catálogo de $\mathrm{SCl}$ de la editorial ELSEVIER, debe hacerlo casi exclusivamente en inglés, formar parte de redes internacionales y pagar, en algunos casos, montos considerables para que pueda ser recibida (no necesariamente publicada), eso sin contar el sesgo de estas revistas hacia los países en desarrollo. Es así que el año 2011, ante la invitación de la administración central, es que se decide crear, en la Universidad Peruana Unión, Tarapoto, la revista Apuntes Universitarios, la misma que tuvo que sortear diferentes trabas administrativas y burocráticas para conseguir su primera publicación. Después de 12 acuerdos de Consejo Universitario, inscripción del nombre legal en los registros de INDECOPY, conseguir el financiamiento respectivo, formar los comités editoriales, comités de redacción y comités científicos asesores, se lanzó la primera edición, basándose en los 33 criterios del catálogo LATINDEX, entre ellos elementos como abstract, keywords, títulos en inglés, cornisas, fechas de recepción y aceptación, leyendas editoriales (con la información pertinente: ISSN, institución editora, abreviatura de la revista, periodicidad, etc.).

Para el año 2012 se consolida la revista, logrando ingresar a Latindex y al Catálogo del Consejo Nacional de Ciencia y Tecnología (CONCYTEC), similar a lo que se hace en Argentina con el Núcleo Básico de revistas, Publindex en Colombia y Qualis en Brasil (Terra-Figari, 2008). 
En la tercera edición, se logra ingresar al catálogo DIALNET, uno de los referentes para toda publicación hispana, asimismo, se implemente el ISSN virtual y el código de barras que es una exigencia en las diferentes ferias internacionales como la Feria Internacional del Libro. También se logra implementar la digitalización completa de la revista a través del repositorio PAPIROS de la UPeU bajo el soporte de DSPACE, y el ingreso Open Journal System (OJS), bajo el dominio: www.apuntesuniversitarios.upeu.edu.pe, logrando mayor visibilidad en la web.

Para el año 2013, con la cuarta edición se logra ingresar al catálogo de GOOGLE ACADÉMICO. Del mismo modo, se aprueba su inclusión en los catálogos de revistas abiertas del Directory of Open Access Journals: DOAJ y Sherpa Romeo. Logrando consolidarse como la primera revista de la UPeU en llegar a dicho catálogo.

Para la quinta edición, se está trabajando fuertemente en la revisión por pares a través de la evaluación "doble ciego", con algunos artículos completamente traducidos al inglés y portugués con el objetivo, a mediano plazo, de llegar a Scielo Perú, Redalyc, ERIC y SCIMAGOR.

\section{Conclusión}

Existe la necesidad de que los investigadores publiquen sus investigaciones en redes de indexación, logrando una mayor visibilidad por parte de la comunidad científica. Asimismo, hemos observado el caso de la revista Apuntes Universitarios y su participación sostenida en sistemas de indexación internacionales; sin embargo, como universidades aún es necesario implementar políticas institucionales que permitan, entre otros aspectos, fomentar la participación de los docentes en revistas indexadas y crear la necesidad en los estudiantes de usar las bases de indexación, que utilicen los repositorios digitales. Asimismo, la creación de la jefatura de publicaciones científicas, en la cual se efectúen procesos de reingeniería de las revistas científicas existentes. Programación continua de cursos-taller de bases de datos en Biblioteca, implementación del curso de redacción científica. El cambio de formato de tesis a formato de artículo científico, instalación del sistema Open Journal System (OJS) para el proceso editorial de las revistas existentes, así como la Instalación del sistema Open Journal System (OJS) para que los alumnos aprendan y practiquen el proceso de envío de manuscrito a una revista que utilice este sistema. 
Josué E. Turpo \& Gerardo E. Medina

\section{Referencias}

Aguirre, J. (2012). Respecto a las Indizaciones e Indexaciones. Revista Científica Ciencia Médica. 15. 1

Ardito, F. (2012). Seminario de Publicación Científica. Asamblea Nacional de Rectores. Pucallpa: Universidad Nacional de Ucayali.

Greenleaf, F. (2009). Historia de la educación adventista. Buenos Aires: Asociación Casa Editora Sudamericana.

Gregorutti, G. (2010). La producción intelectual en pequeñas universidades privadas latinoamericanas: una situación paradójica. Revista Internacional de Estudios en Educación. (10) 1:1-21.

Gregorutti, G. (2011). La producción de investigación en las universidades privadas: estudio de un caso. Revista Enfoques (23) 2: Disponible en Enfoques [online]. 2011, vol.23, n.2 [citado 2013-08-09], pp. 5-20. Disponible en: <http://www.scielo.org.ar/scielo.php?script=sci_ arttext\&pid=S1669-27212011000200002\&Ing=es\&nrm=iso>. ISSN 1669-2721.

Iturriera, S. (2012). Sugerencias para la indexación de revistas de trabajo social como espacios de visibilidad de la productividad académica. Costa Rica: Universidad de Costa Rica. Disponible en: http://www.ts.ucr.ac.cr/binarios/pela/pl-000466.pdfKorniejczuck, R. (2000). Integración de la fe en la enseñanza y el aprendizaje. Montemorelos, México: Publicaciones Universidad de Montemorelos.

Martin, L., Wilson, J. (2002). The Critical Role of Research in Adventist Education. Journal of Adventist Education, 64 (04). pp. 37-40.

Pacheco, J., \& Hurtado, A. (2012). Estrategias para mejorar el impacto de las publicaciones indexadas en Publindex, ISI, Scopues y SciELO: el caso de la Universidad Santo Tomás, Colombia. Revista Hallazgos, 19, 175-191.

Piscoya, L. (2006). Ranking universitario en el Perú, Plan Piloto. Lima: Asamblea Nacional de Rectores.

Rojas, A., Rivera, S. (2012). Guía de buenas prácticas para revistas académicas en acceso abierto. ONG Derechos digitales. Disponible en: http://www.derechosdigitales.org

SIR (2013). SIR Iber 2013 Peru 2007-2011. SCImago Research Group, Copyright 2013. Data Source: Scopus ${ }^{\circledast}$ http://www.scimagolab.com :: http://www.scimagoir.com

Terra-Figari, L. (2008). Diseminación del conocimiento académico en América Latina. Montevideo, Antropología social y cultural en Uruguay, Uruguay, UNESCO.

Top universities (2013). Colleges in Peru 2012 -2013 / las mejores universidades, colegios en el Perú 2012 - 2013. Disponible en: http://www.topstudylinks.com/Top-Universities-in-peru-c95. aspx

Vizcaíno, M. (2011). Indexación: un camino difícil, pero posible. Revista Avances en Enfermería (29) 1: [cited 2013-08-09], pp. 11-12. Available from: <http://www.scielo.org.co/scielo. php?script=sci_arttext\&pid=S0121-45002011000100001\&lng=en\&nrm=iso>. ISSN 01214500 . 